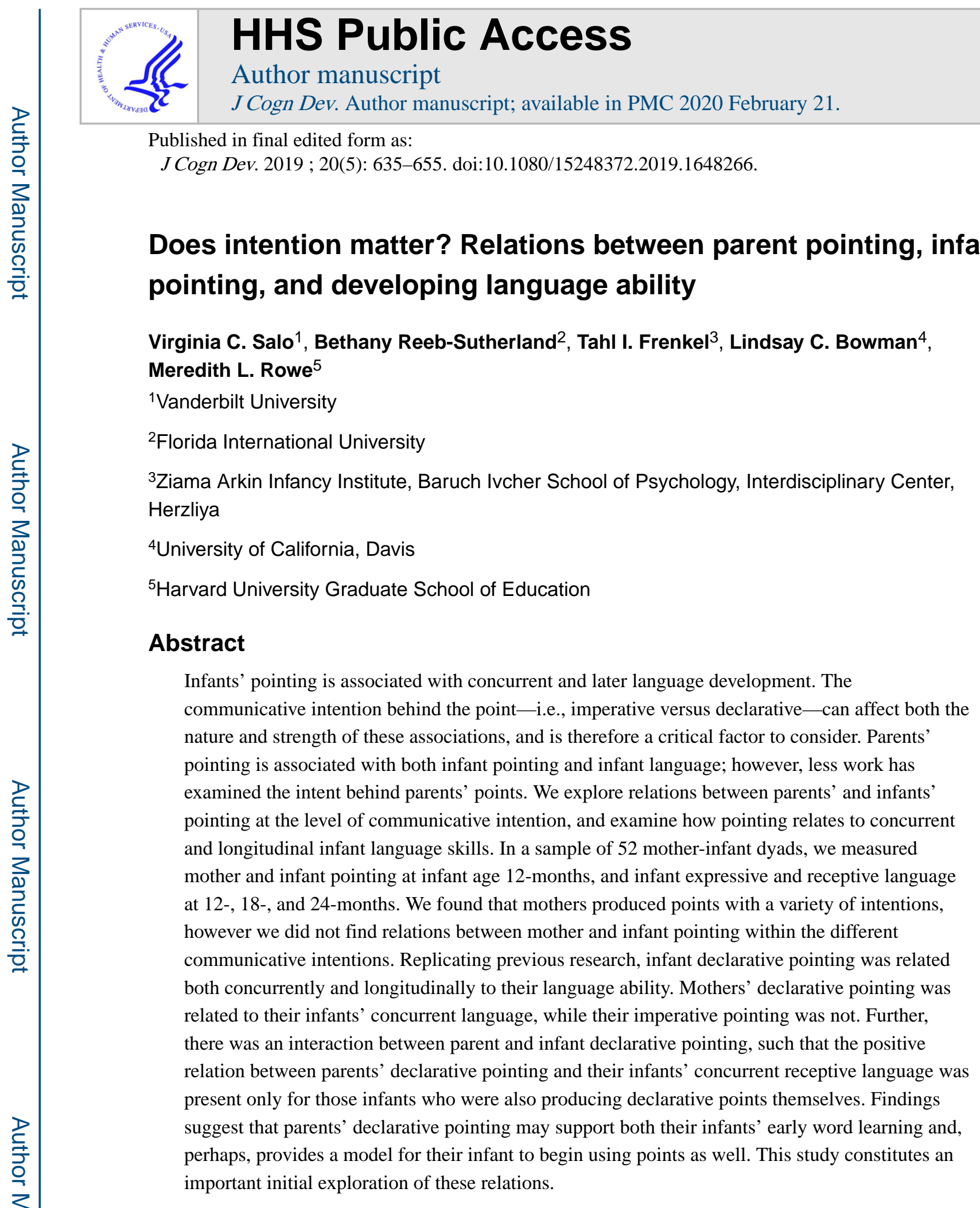

Infant's pointing production toward the end of the first year of life is an early marker of emerging communicative skill and a predictor of later language ability (Colonnesi, Stams, Koster, \& Noom, 2010; Goldin-Meadow, 2007; Tomasello, Carpenter, \& Liszkowski, 2007).

Address for contact: Virginia C. Salo, Department of Psychology and Human Development, Peabody College \#552, 230 Appleton Place, Nashville, TN 37203 (virginia.salo@vanderbilt.edu). 
Infants produce points with a variety of communicative intentions (Begus \& Southgate, 2012; Knudsen \& Liszkowski, 2013; Liszkowski, Carpenter, Henning, Striano, \& Tomasello, 2004; Liszkowski, Carpenter, Striano, \& Tomasello, 2006). Declarative points, those that are produced with the communicative intention of sharing attention to an object or event, or expressing emotion or information about an object or event, are of particular interest as these types of points are considered an advanced form of pointing and predict later language skill (Bates, Camaioni, \& Volterra, 1975; Colonnesi et al., 2010; but see also Southgate, Van Maanen, \& Csibra, 2007). Additionally, there is a documented link between parents' pointing and their children's own pointing production and language development (Liszkowski, Brown, Callaghan, Takada, \& de Vos, 2012; Liszkowski \& Tomasello, 2011; Matthews, Behne, Lieven, \& Tomasello, 2012; Rowe, 2000; Rowe \& Goldin-Meadow, 2009a; Salomo \& Liszkowski, 2013; Rowe \& Leech, under review), however this literature has largely ignored the communicative intent behind parents' pointing focusing instead on more global measures such as the number of points a parent produces or the number of different meanings produced with points. It is therefore unknown whether individual differences in infants' production of points with different communicative intentions may be influenced by the communicative intents of their parents' points. As it is such an important social-communicative milestone, it is important to understand the various factors that may influence the development of pointing. Our aim in the current study was thus to begin addressing this gap in the literature by examining variation in the communicative intents of the points that parents produce with their infants. Further, we explored whether individual differences in the communicative intentions behind parents' pointing with their infants are related to the type of points that their infants produce and/or to their infants' developing language abilities.

\section{Infant Pointing}

Infants communicate through gestures before they start speaking, and infants' pointing gestures are of particular interest as a precursor to later language skills. Soon after they emerge around 10-12 months, points quickly become the most common gesture that infants produce (Bates et al., 1975; Camaioni, 1997; Carpenter, Nagell, \& Tomasello, 1998; Franco \& Butterworth, 1996; Lock, Young, Service, \& Chandler, 1990), and infants' production of pointing is closely linked with developing language skills (Colonnesi et al., 2010). The age of onset of pointing predicts vocabulary growth over the second year of life (Brooks \& Meltzoff, 2008; Desrochers, Morissette, \& Ricard, 1995), infants who point more and to a greater number of different objects during interactions with caregivers have stronger language skills in kindergarten (Rowe \& Goldin-Meadow, 2009b), and using a point in conjunction with a word predicts the production of two-word combinations (Iverson \& Goldin-Meadow, 2005). In children with traumatic brain injury, delays in pointing onset also predict delayed language acquisition (Sauer, Levine, \& Goldin-Meadow, 2010).

Intriguingly, some evidence suggests that infants may use points to convey different messages to a communicative partner, and the different types of points infants use may also signal different abilities of the infant. In general, pointing is a flexible gesture in that it can be used to convey a variety of different messages to a communicative partner. Points are considered under the umbrella of deictic gestures - gestures that indicate a referent and the 
meaning of which is thus dependent on the context (Cartmill, Demir, \& Goldin-Meadow, 2012). That is, the meaning of a point can change depending on what is being pointed to as well as the joint understanding created around that object in the moment, as compared to a conventional gesture, like a head nod, whose meaning is inherent in the gesture itself and is constant across contexts. Important for the current study, the single gesture form of pointing can convey a variety of different communicative intentions. For example, one might point to a toy that is out of reach to request a partner to hand it over. Alternatively, one might point to that same toy in response to a partner's asking for its location. The two main categories of communicative intentions that capture these differences are imperative and declarative. Imperative points are used to control a partner's behavior, as in the first example above wherein the goal of the interaction is to get the toy and the partner is being used as a tool to achieve that end. Declarative points ${ }^{1}$, on the other hand, are used to share information and attention with a partner to an object or event, as in the latter example above.

Research demonstrates that infants engage in both imperative and declarative points (Cochet $\&$ Vauclair, 2010). Declarative pointing is hypothesized to be a more advanced form of pointing because it emerges later in development (Bates et al., 1975), and is proposed to require an understanding of one's partner as a mental agent, whereas imperative pointing is proposed to only require an understanding of one's partner as a causal agent (Camaioni, 1997; although see Tomasello et al., 2007 for a more rich interpretation of both declarative and imperative gestures). While an imperative point may indicate an understanding of a partner's attention or knowledge state, production of these points may also rely merely on a more basic understanding that your partner can be used as a tool to achieve your goal. Declarative pointing, in which the goal itself is to share attention or information, is considered a better indicator that the infant in fact understands their partner as having an attentional or knowledge state and is aware of her own ability to manipulate that attention/ knowledge state (Liszkowski, Carpenter, \& Tomasello, 2008). Importantly, declarative pointing is also a stronger predictor of later language ability, as compared to imperative pointing (Colonnesi et al., 2010; although see Lüke, Grimminger, Rohlfing, Liszkowski, \& Ritterfeld, 2017). For example, Desrochers and colleagues (1995) found that infants who produced declarative points earlier than their peers tended to have stronger language skills later, whereas that same comparison did not hold for imperative pointing. Tomasello, Carpenter, and Liszkowski (2007) have argued for identifying two subtypes of declarative pointing: expressive, wherein the pointers' intention is to share an emotional reaction about a referent, and informative, wherein the pointers' intention is to share information about a referent she thinks her partner will find of interest. To our knowledge, no empirical work has yet examined individual variability in the use of these two subtypes of declarative points.

In a series of studies with 12-month-olds, Liszkowski and colleagues found evidence that infants use pointing gestures flexibly with a variety of intentions, and that they are able to

\footnotetext{
${ }^{1}$ While recent studies have suggested that declarative pointing may alternatively reflect interrogative intentions during which infants' points to an object are primarily used to obtain information from a partner about that object (Harris \& Lane, 2014; Kovács, Tauzin, Téglás, Gergely, \& Csibra, 2014; Southgate et al., 2007), the current study was unable to examine this directly given the study design. Therefore, the more commonly used definition of declarative pointing was used to refer to a more advanced form of pointing in contrast to imperative pointing. Additionally, declarative as compared to interrogative pointing is more commonly expressed in caregivers making it a common measure that can be examined in both infants and their caregivers.
} 
reliably produce declarative points at this age (Knudsen \& Liszkowski, 2013; Liszkowski et al., 2004, 2006, 2008). However, other studies have shown significant variation in infants' production of both imperative and declarative points around 12 months (Brooks \& Meltzoff, 2008; Lüke et al., 2017; Mundy et al., 2007; Salo, Rowe, \& Reeb-Sutherland, 2018). It is still unclear what factors predict these individual differences. Such clarity is important given the links between pointing and critical infant linguistic milestones (e.g., Cochet \& Byrne, 2016). Indeed, elucidating aspects of the infants' environment that may support infants' pointing, and declarative pointing in particular, could have implications for better understanding the individual variation that exists in infants' preverbal communicative skills as well as for identifying appropriate and effective interventions for those infants at risk for language delay. As we discuss next, one promising candidate that may influence infant pointing production is parents' own pointing.

\section{Parent Pointing}

Whether and how variations in the intentions behind parents' pointing might foster infants' pointing, or infant language, has yet to be examined, however parents' pointing is positively related to their infants' pointing when measured regardless of intentions. That is, parents who produce more points have infants who also produce more points during interaction (Liszkowski \& Tomasello, 2011; Rowe, 2000; Rowe \& Goldin-Meadow, 2009a; Salomo \& Liszkowski, 2013). Further, parent pointing is positively related to their infant's language ability (Rowe, 2000). These empirical associations are supported by training studies that provide experimental evidence that infants' pointing is related to the points they observe in their environment. Two training studies have specifically targeted parents, aiming to indirectly affect infant pointing production through increasing parents' use of points. Matthews and colleagues (2012) compared 9- to 11-month-old infants pointing behavior before and after a parent-focused training on pointing. Parents in the training group were instructed to practice demonstrating pointing for their infants over a month-long period. There was also a control group in which parents were given instructions to practice nursery rhymes and play with musical instruments with their infant. While the intervention was not associated with an effect on overall amount of pointing production or on the age of emergence of pointing, both exposure to training and frequency of maternal pointing were found to predict infants' gaze-checking while pointing, that is monitoring where their partner is looking while producing a pointing gesture. Gaze-checking while pointing is considered an indicator that infants' are indeed pointing with declarative communicative intent and thus demonstrating an understanding of their partner's attentional state (e.g., Bates et al., 1975). These findings suggest that observational experience of points (via the parent) may potentially influence an infants' understanding of how pointing can be used to manipulate the attention state of others. In a similar study, Rowe and Leech (2018) trained parents of 10-month-olds on the importance of pointing with their infants, the role pointing plays in language development, and how they can make a difference in their infants' development by pointing with them often. Parents in the training group were provided with toys and told to play and point for 15 minutes a day with their infant. Parents in the control group were not given the information about pointing, and were also given the toys and told to play for 15 minutes a day. At a follow-up home visit 2 months later, parents in the 
treatment group pointed more and to a greater number of different objects during play with their 12-month-olds than parents in the control group. Further, the infants of parents in the treatment group pointed more and to a greater number of different objects during play than the infants of parents in the control group. However, the effects on infant pointing production were not sustained in subsequent follow-ups at 14-, 16-, and 18-months. Interestingly, while there was no main effect of the intervention on infants' vocabulary growth over the course of the study, the authors did find an effect for a subset of the participants whose parents had endorsed a fixed mindset of intelligence at the start of the intervention. These parents exhibited a stronger effect of the training on their own pointing, and their infants exhibited greater pointing at 12-months, and greater vocabulary growth from 10- to 18-months. Thus, there is both correlational and experimental evidence suggesting that infants' pointing (either in terms of sophistication or frequency) may be fostered by parents' own pointing production, and that individual differences on the part of parents are important to consider.

Another training study compared the effects of manipulating infants' observed or own experience pointing. LeBarton and colleagues (2015) randomly assigned 17-month-olds to one of three six-week in-home training conditions: one in which an experimenter modeled pointing and labeling with the infant and also encouraged the infant to point, one in which the experimenter pointed but did not encourage the infant to point, and one in which the experimenter did not point nor did she encourage the infant to point. Across the training period, infants in the experimenter and infant pointing condition produced more points. This increase in infant gesture was associated with an increase in parents' gesture use (although parent gesture was not a focus of the study), and, importantly, with group differences in infants' productive vocabulary at the end of the six-week period. However, they did not see a similar effect on either pointing production or vocabulary for infants in the condition in which only the experimenter pointed without encouraging the infant to point. The results of this study provide more evidence for an association between parents' and infants' pointing, but also suggest that infants' own pointing may be particularly supportive for their language development.

Taken together, these findings suggest that infants' pointing can be influenced by parent use of pointing, but that this is not always the case. It is important to note that the bulk of the research in this area has not examined the intentionality behind the points that parents are producing with their infants. Doing so may help to explain some of the nuance in the extant literature, and may help to elucidate the role of parents' pointing in supporting both infant pointing and infant language development. One exception is a study by Rowe (2000) with a low-income sample of mothers and their 14-month-old infants which found that the majority of mothers' points were produced to direct their infants' attention to something $(66 \%$, $\mathrm{M}=10.0, \mathrm{SD}=8.8$ ), as opposed to being produced in the context of discussing an object of shared attention $(19 \%, \mathrm{M}=2.9, \mathrm{SD}=3.5)$ or for negotiating the activity $(15 \%, \mathrm{M}=2.2$, $\mathrm{SD}=2.9)$. Directing attention and discussing an object of shared attention may be interpreted as indexing declarative intention, and negotiating the activity as imperative. This study provides initial evidence that mothers use more declarative than imperative points with their infants during book reading and toy play, yet the large amount of observed variability in these behaviors warrants further investigation. It is possible that infants who observe their 
parent(s) using more points in the context of sharing attention, emotion, and information about objects or events are more likely to understand and use these kinds of points themselves. However, this potential mechanism for the emergence of declarative pointing has yet to be examined.

Parent pointing might also be related more directly to infant language development. Synchronizing their gesture use with talk about an object or event signals to the infant that these two are linked and directs the infant's attention to the referent, thereby narrowing the problem space for the infant and making it easier to map the spoken label onto the referent (de Villiers Rader \& Zukow-Goldring, 2012). Indeed, there is evidence that infants learn better when talk is centered on the things that are most prominent in their attention and that they are focusing on in their environment (Yu \& Smith, 2012). It is possible that different types of pointing gestures, with different communicative intentions, might be more or less likely to draw the infant's attention. Differential relations between parents' use of certain types of intentions with their pointing gestures and their infants' language development would support this idea.

In summary, by the end of their first year, infants produce points with a variety of communicative intentions (e.g., Liszkowski et al., 2004, 2008) and while pointing overall is a predictor of their growing language skills (e.g., Rowe \& Goldin-Meadow, 2009b), declarative pointing is a stronger predictor than imperative pointing (Colonnesi et al., 2010). Additionally, parents' overall pointing production is related to their infants' pointing (Liszkowski \& Tomasello, 2011; Rowe, 2000), and increasing parent pointing can lead to increases in infant pointing (LeBarton et al., 2015; Rowe \& Leech, 2018). However, it is unknown whether parent declarative versus imperative points might differentially relate to the kinds of points their infants produce or to their infants' language development. Examining these relations may help determine whether specific types of parent pointing are important for the development of specific types of infant pointing which may in turn lead to a better understanding of the involvement of parent pointing in language development.

\section{The current study}

The aim of the current study is to examine the relations between parents' pointing and infants' pointing at the level of the communicative intention, and infants' developing language skills, in order to better understand how the early environment may shape infants' communicative development. In particular, because infant declarative pointing seems to be a stronger predictor of language development (Colonnesi et al., 2010), we focus on the role of parent pointing in supporting infants' declarative pointing. We hypothesize that parents' declarative pointing will relate to their infants' own declarative pointing. Based on the stronger relations between infants' own declarative pointing and their language development, we also predict that parents' declarative pointing will be a stronger predictor of their infants' language skills than parent's imperative pointing. Lastly, we examine how infants' and parents' pointing compare as predictors of the infants' developing language skills. In previous research, infant pointing has been shown to mediate the relation between parent pointing and infant language (e.g., Rowe \& Goldin-Meadow, 2009a). We explore this possibility, predicting that a similar trajectory will hold at the level of communicative intent 
such that infants' declarative points may mediate any relations between parent declarative points and infant language outcomes. However, given the specificity of the type of pointing, it is also possible that infants' declarative pointing might moderate rather than mediate a relation between parent declarative pointing and infant language skills. There is evidence that infants' production of points is related to their understanding of the same gesture (Behne, Liszkowski, Carpenter, \& Tomasello, 2012; Liszkowski \& Tomasello, 2011; Woodward \& Guajardo, 2002), and the same seems to be true specifically for declarative points (Salo et al., 2018). Thus, perhaps infants who are already producing declarative points, and thus displaying an early behavioral marker of symbolic communication and intention understanding, are better able to understand the significance of others' declarative points and use those observed declarative points to support them in learning new words. Therefore, we also test for moderation effects to compare the above hypotheses and examine how parents' and infants' declarative pointing compare as predictors of the infants' developing language skills.

In sum, our specific research questions were:

1. How does parents' production of points vary by communicative intent? That is, do parents tend to use points with specific intents more than others when interacting with their infant?

2. Do the relations between parent pointing and infant declarative pointing differ based on the communicative intent of parents' points?

3. What are the relations between parent pointing, within each type of communicative intent, and their infants' developing language skills?

4. How do parent and infant declarative pointing compare as predictors of infant language skills?

\section{Methods}

\section{Participants}

The current sample was drawn from a larger sample of 124 infants participating in a longitudinal study starting at 1 month through 24 months (Reeb-Sutherland, Levitt, \& Fox, 2012; Salo et al., 2018). Families were recruited from counties surrounding a large midAtlantic university. For the current analyses we used data from the 12-month, 18-month, and 24-month visits. Of the original 124 infants seen at 1 month, 41 did not return to participate in the 12-month visit. An additional 28 dyads were excluded from analysis for the following reasons: 22 dyads did not participate in the parent-infant interaction, and six mothers did not speak English during the parent-infant interaction (3 Spanish, 2 Chinese, 1 Korean). Prior to analysis, outliers were identified if they had a standardized score greater than $\pm 3 \mathrm{SD}$. Three cases had extreme values and were removed from the analysis sample, thus the final sample at 12-months included 52 dyads. Of that, 46 dyads completed the 12-month language measure, 44 completed the 18-month language measure and 47 completed the 24-month language measures. 
Out of the 52 dyads included in the 12-month analyses, 24 of the infants were female. Most mothers had a Bachelor's or graduate degree $(n=42), 1$ mother had an Associate's degree, 8 had a high school diploma or below, and 1 mother did not report her education level. Thirtythree mothers self-identified as Caucasian, 11 as African-American, 3 as Asian, 3 as Hispanic and 2 as other. At the 12-month visit, infants were on average 12.22 months old (Range $=11.90-12.63 ; \mathrm{SD}=.19)$. At the 18-month follow-up, 19 of the infants were female and average age was 18.18 months (Range $=17.67-19.30, \mathrm{SD}=.37$ ). At the 24month visit, 23 of the infants were female and average age was 25.38 months (Range = $24.40-26.30, \mathrm{SD}=.50$ )

\section{Procedure}

During the 12-month visit, dyads took part in a 10-minute videotaped, free play interaction. During these interactions, dyads were provided an assortment of age-appropriate toys, including a picture book, farm set, and shape sorter. Parents were instructed to play with the toys with their infant as they normally would, but to try to play at least once with each of the three objects. These interactions were then transcribed and coded for pointing gesture production (methods described in detail below). In addition, at infant age 12-, 18-, and 24months, mothers reported on their infants' expressive and receptive language skills.

\section{Transcription and coding}

All speech and gestures during the mother-infant videotaped interactions at 12 months were transcribed by research assistants trained to transcribe reliably using the CHAT conventions (https://talkbank.org/manuals/CHAT.pdf) of the Child Language Data Exchange System (CHILDES; MacWhinney, 2000). The unit of transcription was the utterance, bounded by grammatical closure, a pause of more than two seconds, or transition in speaker.

Transcription reliability was established by having a second reliable coder transcribe $20 \%$ of the videotapes; reliability was assessed at the utterance level and was achieved when coders agreed on $95 \%$ of transcription decisions.

\section{Measures}

Parent and infant pointing production.-Parent and infant points were coded from the transcripts and videos of the mother-infant 10-minute free play interaction task that took place during the 12-month visit. Points were defined as any use of the extended index finger or open palm to indicate something in the environment. Open hand points were coded if only one arm was extended and the infant was not leaning forward or otherwise apparently attempting to reach for the referent (Franco \& Butterworth, 1996). Only infants produced open hand points. If a parent or infant had their finger extended into a point shape, but it was not communicative (e.g., using the index finger to push a button on a toy) it was not coded as a pointing gesture. Point tokens, the total number of pointing gestures (e.g., Goldin-Meadow, Mylander, de Villiers, Bates, \& Volterra, 1984), served as a measure of the quantity of parent and infant overall pointing production, regardless of intention. To address the research questions outlined above regarding relations between parent and infant pointing across the various types of communicative intentions, we further coded both parents' and infants' pointing gestures according to their intention. Points were categorized first according to 
whether they were declarative or imperative. Declarative points were defined as those intended to direct the partner's attention and to share information or emotion about an object or event, whereas imperative points were defined as those intended to elicit a specific action from their partner or to use their partner as a means towards a non-social end goal (e.g., to retrieve an object; Bates et al., 1975). Based on Tomasello and colleagues' (2007) theoretical description of two subtypes of declarative points, described above, these were further broken down into expressive declarative - pointing to share emotion about an object or event (often surprise or delight) - or informative declarative - pointing to share information about an object or event (e.g., the location or a label). Intention was derived from the context surrounding the pointing gesture, including any associated verbalizations or speech, body language, and partner's response. Reliability between two independent coders was assessed for $10 \%$ of the free-play interactions. Agreement between coders was $98 \%(\mathrm{k}=.93, \mathrm{~N}=206)$ for identification of points, and $87 \%(\mathrm{k}=.78 ; \mathrm{N}=137)$ for communicative intention of pointing gestures.

As our research questions focus specifically on infants' declarative pointing, only infants' declarative points are included in the current study, however all categories of parents' points are included in our analyses. Descriptive statistics for parent and infant pointing production are shown in Table 1. Approximately half of the infants produced one or more declarative point during the 12-month interaction $(n=25)$. Only one infant produced a single informative declarative point across all the interactions. Therefore, only the superordinate declarative category is considered for infants in the following analyses, however these can be considered as mostly representing infants' use of expressive declarative points. As reviewed above, infants' ability to produce declarative points is related to their understanding of declarative points (Salo et al., 2018), and infants' ability to produce declarative points earlier than their peers predicts later language ability whereas the same is not true for imperative points (Desrochers et al., 1995). As such, we recalculated infants' declarative pointing as a dichotomous measure with a score of 1 indicating that the infant did produce a declarative point $(\mathrm{N}=25)$, and a score of 0 indicating that the infant $\operatorname{did}$ not $(\mathrm{N}=27)$.

Infant language.-To maintain consistency in method of infant language measures, parent report measures were utilized at all three time-points. Infants' expressive and receptive vocabulary skills were measured at 12- and 18-months respectively via parent report using MacArthur-Bates Communicative Developmental Inventories, Words and Gestures (MCDIWG; Fenson et al., 1994). The vocabulary section of the MCDI-WG provides a checklist of 396 words for parents to indicate whether their infant understands or understands and uses each word. The total number of words a parent indicates their infant understands serves as a measure of their infant's receptive vocabulary (12-months: $\mathrm{M}=83.02, \mathrm{SD}=74.26$; 18 -months: $\mathrm{M}=213.70, \mathrm{SD}=92.07)$. The total number of words a parent indicates their infant understands and produces serves as a measure of their infant's expressive vocabulary (12months: $\mathrm{M}=7.33, \mathrm{SD}=6.65$; 18-months: $\mathrm{M}=76.48, \mathrm{SD}=62.94$ ).

At infant age 24-months, expressive and receptive language ability was measured via two different parent report surveys. For consistency with the previous time points, we report scores on the MacArthur-Bates Communicative Development Inventory, Words and Sentences (MCDI-WS; Fenson et al., 1994), which provides a checklist of 680 words for 
parents to indicate whether their infant produces each word $(\mathrm{M}=365.74, \mathrm{SD}=172.70)$. At 24months the MCDI-WS does not offer a measure of receptive language. Therefore, we also had parents complete the receptive and expressive language subscales of the Vineland Adaptive Behavior Scales $2^{\text {nd }}$ edition (Vineland-II; Sparrow, Cicchetti, \& Balla, 2005). The receptive language subscale asks questions about infants' ability to respond appropriately to various kinds of verbal input and instructions. The expressive subscale asks questions about infants' vocabulary size and the complexity of the language they use. The Vineland-II provides standardized scores that were used in the current study (Receptive: $\mathrm{M}=16.55$, $\mathrm{SD}=1.82$; Expressive: $\mathrm{M}=16.36, \mathrm{SD}=1.52$ ).

Mothers' language.-Two measures of mothers' spontaneous language produced during the free-play interaction were extracted from the transcripts. Word tokens, or the total number of words spoken, is a measure of quantity of speech $(\mathrm{M}=612.62, \mathrm{SD}=268.26)$. Word types, or the total number of unique words spoken, is a measure of the diversity of speech $(\mathrm{M}=147.02, \mathrm{SD}=36.73)$. As mothers' speech to their infants is a significant predictor of infants' developing language skills (Hart \& Risley, 1992, 1995; Huttenlocher, Haight, Bryk, Seltzer, \& Lyons, 1991; Pan, Rowe, Singer, \& Snow, 2005; Rowe, 2012) and there is evidence that mothers who point more also talk more (Rowe, 2000), we wanted to include measures of mothers' speech to ensure that any relations seen between mothers' and infants' pointing and language were not being driven by mothers' speech.

\section{Results}

To fully explore our research questions, we set alpha at the conventional $\mathrm{p}<0.5$ and did not correct for multiple comparisons. Further, to provide a comprehensive picture of the results, we also consider trends at $\mathrm{p}<.1$.

\section{Parent pointing}

Our first goal was to examine and compare the patterns of mothers' production of points across the various categories of communicative intentions. Mothers produced more declarative points than imperative points on average $(\mathrm{t}(51)=3.88, \mathrm{p}<.001)$. When considering the declarative pointing subcategories, mothers produced significantly more expressive than informative declarative points $(\mathrm{t}(51)=4.03, \mathrm{p}<.001)$.

\section{Relations between parent and infant pointing}

We next examined whether the relations between parent and infant pointing varied within the different types of communicative intentions. We ran a series of zero-order Pearson correlations between the parent pointing measures and the total number of point tokens their infants produced. None of these relations were statistically significant (all r's <.17, all p's $>$.23). Next, we conducted independent samples t-tests to compare mothers' production of points across the dichotomous infant declarative pointing groups. Mothers of infants who did produce declarative points had a tendency to produce more total declarative points and more expressive declarative points (Table 2), however these comparisons did not reach significance. 


\section{Relations between parenting pointing and infant language}

Next, we examined the relations between parent pointing and infant language. We

hypothesized that mothers' declarative points might be more strongly related to their infants' developing language skills than their imperative pointing. Interestingly, we found that this hypothesis was supported, but primarily in relation to infants' language skills at 12-months, not later. As shown in Table 3, mothers' overall point tokens was strongly and positively correlated with their infants' receptive vocabulary at 12 -months ( $p<.01)$. Importantly, this relation seems to be specific to mothers' declarative pointing as mothers' expressive declarative and informative declarative points were both significantly positively correlated with their infants' receptive vocabulary at 12 months, and mothers' imperative points were not. However, at 18-months, only mothers' expressive declarative points were related to their infants' receptive vocabulary. When controlling for infants' 12-month receptive vocabulary, this relation was no longer significant $(\mathrm{r}=-.02, \mathrm{p}=.89)$. There were no significant relations between mothers' imperative points and their infants' language skills. There were also no significant relations with any of the parent pointing measures and the 24-month language measures (Table 4). In sum, relatively strong relations are seen between parent pointing, particularly declarative pointing, and infants' receptive language skills at 12-months, but these relations are not evident at 18- or 24-months.

\section{Comparing parent and infant pointing as predictors of infant language}

Relations between infant pointing and infant language.-Prior to addressing our fourth research question comparing parent and infant pointing as predictors of infant language, we first looked at whether infants' pointing was related to their concurrent and later language ability. We first report relations between infant total number of points and language, then look at relations between declarative pointing status and language. The total number of points infants produced at 12-months was not correlated with their 12-month language scores (Receptive: $\mathrm{r}=.19, \mathrm{p}=.21$; Expressive: $\mathrm{r}=.04, \mathrm{p}=.81$ ); however infant total points at 12-months was moderately correlated with their 18 -month receptive vocabulary $(\mathrm{r}=.28, \mathrm{p}=.07)$ and significantly correlated with their 18-month expressive vocabulary ( $\mathrm{r}=.37$, $\mathrm{p}=.01$ ). The relation with 18 -month receptive vocabulary was non-significant when controlling for 12-month receptive vocabulary $(\mathrm{r}(\mathrm{par}, d f=38)=.23, \mathrm{p}=.16$, however the relation with 18-month expressive vocabulary remained significant after controlling for either 12-month receptive vocabulary $(\mathrm{r}(\mathrm{par}, d f=38)=.33, \mathrm{p}=.04)$ or 12 -month expressive vocabulary $(\mathrm{r}(\mathrm{par}, d f=38)=.41, \mathrm{p}=.009)$. The relation between infant total points and 24month expressive MCDI scores was not significant $(\mathrm{r}=.14, \mathrm{p}=.35)$. However, the relations with both receptive and expressive Vineland scores were significant (Receptive: $r=.33$, $\mathrm{p}=.02$; Expressive: $\mathrm{r}=.32, \mathrm{p}=.03$ ), and remained so after controlling for the 12-month receptive vocabulary $(\mathrm{r}(\mathrm{par}, d f=39)=.37, \mathrm{p}=.02)$ and 12 -month expressive vocabulary ( $\mathrm{r}(\mathrm{par}$, $d f=39)=.34, p=.03$ ), respectively. In sum, infants who pointed more during the 12-month interaction, had larger MCDI expressive scores at 18 months, and larger receptive and expressive Vineland scores at 24 months, controlling for their 12-month language scores.

As can be seen in Table 4, infants who produced any declarative points at 12-months had stronger MCDI receptive scores at that same time ( $\mathrm{p}<.05)$, moderately stronger receptive and expressive MCDI scores at 18-months $(\mathrm{p}<.10)$, and stronger Vineland scores at 24-months, 
particularly expressive scores $(\mathrm{p}<.05)$. We conducted a series of regression models predicting infants' language skills at 18- and 24-months from their declarative pointing status, while also controlling for 12-month receptive language. The relations with 18-month vocabulary were non-significant with the added control (Receptive: $\beta=.09, \mathrm{t}=.64, \mathrm{p}=.53$; Expressive $\beta=.18, t=1.17, p=.25$ ). Declarative pointing status became a significant predictor of 24-month Vineland receptive language when controlling for 12-month receptive vocabulary ( $\beta=.34, t=2.20, p=.03$ ). Lastly, the significant relation between declarative pointing status and 24-month vineland expressive vocabulary was maintained after controlling for 12-month language ( $\beta=.39, \mathrm{t}=2.45, \mathrm{p}=.02)$. In sum, infants who produced declarative points at 12-months had stronger concurrent receptive language scores, and stronger 24-month language scores even when controlling for 12-month language scores.

Given results of the above analyses demonstrating links between both parent pointing and infant pointing and infants' language skills, we then examined more complex models predicting infant language that included both parent and infant pointing as predictors. To compare mothers' and infants' pointing as they relate to infants' language ability, we conducted a series of regression analyses predicting infants' receptive vocabulary at 12months, concurrent to the pointing measures. Specifically, we compared mothers' expressive declarative points and infants' declarative pointing status as predictors of infant receptive vocabulary at 12 -months. We conducted both a mediation model, wherein infant pointing was examined as a mediator for the relation between mothers' pointing and infant language, and a moderation model, wherein we examined the interactive effects of parent and infant pointing. Variables were mean-centered before being entered into the models.

Table 5 shows the results of the regression analyses with 12-month receptive vocabulary as the outcome variable. When considered separately, both mothers' expressive declarative points and infant declarative pointing status are significant predictors, as can be seen in Models 1 and 2. In Model 3, which tests for a mediation effect by considering both mother and infant pointing together, the parameter for infant declarative pointing status is no longer significant, whereas mother's production of expressive declarative points remains a significant predictor of their infants' concurrent receptive vocabulary. Thus, infant pointing status is not a mediator between mother and infant language, and the results suggest collinearity amongst the parent and infant pointing measures. We next examined a potential moderation by entering the interaction between mother expressive declarative pointing and infant declarative pointing status into the model (Model 4). The interaction parameter was significant and adding the interaction term significantly improved the model fit $(\mathrm{F}(1,42)=14.20, \mathrm{p}=.001)$. Analysis of simple slopes showed that the positive relation between mothers' expressive declarative pointing and their infants' concurrent receptive language is significant only when infants were also producing declarative points $(B=7.85$, $\mathrm{p}<.001$; Figure 1). For infants who did not produce declarative points, the relation between mothers' pointing and their language was not significant $(\mathrm{B}=-1.37, \mathrm{p}=.48$; Figure 1).

\section{Examining the potential role of mothers' speech}

As mentioned above, measures of mothers' speech to their infants during the free-play interaction were extracted. Although this was not a central question for the current study, 
these measures were compared with mothers' pointing to ensure that the relations shown above between mothers' and infants' pointing and language were not being driven by mothers' speech. Indeed, mothers' language use during the 12-month free-play interaction was positively correlated with their pointing during the interaction. Specifically, mothers who produced more word tokens also produced more total points $(\mathrm{r}=.30, \mathrm{p}=.03)$ and expressive declarative points $(\mathrm{r}=.31, \mathrm{p}=.02)$, and produced marginally more total declarative points $(\mathrm{r}=.27, \mathrm{p}=.06)$ and imperative points $(\mathrm{r}=.23, \mathrm{p}=.10)$. Greater number of maternal word types was significantly positively related to expressive declarative points $(r=.30, p=.03)$. However, mothers' speech was not related to their infants' pointing. That is, there was no relation between mothers' word types and tokens and total infant points produced (Word Types: $\mathrm{r}=.03, \mathrm{p}=.86$; Word Tokens: $\mathrm{r}=-.04, \mathrm{p}=.80$ ), and there were no group differences in mothers' speech across the dichotomous measure of infants' declarative pointing (Word Types: $\mathrm{t}(50)=-0.56, \mathrm{p}=.58$; Word Tokens: $\mathrm{t}(50)=0.05, \mathrm{p}=.96)$. The only relation between maternal speech and infant language was between maternal word types and 24-month receptive language ( $\mathrm{r}=.26, \mathrm{p}=.06)$, which was marginal. When controlling for either mothers' word types or word tokens, the relations between mother and infant pointing and between mothers' pointing and infant language all remain unchanged in terms of significance. The tendency for mothers of infants who did produce declarative points to produce more total declarative points and more expressive declarative points still did not reach significance (p's>.05). The relations between mothers' overall point tokens, declarative points, expressive declarative points and informative declarative points, and their infants' receptive vocabulary at 12 -months all remained significant (p's<.01).

\section{Discussion}

The current study adds to the existing literature by exploring the relations between parents' and infants' pointing at the level of communicative intention. We were particularly interested in whether infants' exposure to different communicative intents through their parents' pointing gestures might be associated with different uses of the pointing gestures themselves and related to language development. In this way, we sought to further unpack the factors contributing to infants' language development, and to better characterize the role that parent and infant pointing may play in supporting this development. We began with four main research questions addressing 1) the extent to which parents' production of points varies by communicative intention, 2) whether the relations between parent pointing and infant declarative pointing vary by communicative intention, 3) whether the relations between parent pointing and infant language development vary based on the type of point, and 4) how parent and infant pointing compare as predictors of infant language when measured at the level of the communicative intention. In sum, we found that the mothers in our sample were producing points with a variety of intentions, however we did not find relations between mother and infant pointing within the different communicative intentions. Replicating previous research (Colonnesi et al., 2010), we found that infant declarative pointing was related concurrently and longitudinally to their language ability. We also found that mothers' declarative pointing was related to their infants' concurrent receptive language, while their imperative pointing was not. Most intriguingly, we found an interaction between mothers' expressive declarative points and infants' declarative pointing status, suggesting a nuanced 
relation between parent and infant declarative pointing and infant concurrent receptive vocabulary. We discuss each of these findings below.

\section{Variability in parent pointing intentions}

We did find variability in the types of communicative intentions mothers conveyed through the points they produced in interaction with their infants. Specifically, we found that mothers produced more declarative than imperative points. We also found that parents produce more expressive than informative declarative points. That is, mothers were more often using their pointing gestures to convey information or to share emotion with their infants, as compared to directing their infant to do something, and further they were more likely to point in order to share emotion rather than to convey information. Thus, just as has been observed in infants, mothers in this study were producing points with a variety of communicative intentions and there was variability in the amount of each type of point that mothers were using. This was the first study to directly address whether such variability exists in the kinds of points parents produce. It was necessary to establish such variability before examining whether nuanced relations existed between parent pointing and infant pointing, or between parent pointing and infant language, at the level of communicative intent.

\section{Relations between parent and infant pointing}

While the relations were not statistically significant, we did find trend-level evidence suggesting that the relation between mothers' and infants' pointing varies within the different intentions. Surprisingly, we did not find a relation between mothers' and infants' total number of points, a relation that has been found in previous research (e.g., Liszkowski et al., 2012; Rowe \& Goldin-Meadow, 2009a). This might be explained by differences in the methodology for the parent-infant interaction. For example, previous studies have provided a more dynamic room with objects and decorations displayed in a museum type setup (Liszkowski et al., 2012), or have conducted the interactions in the participants providing fewer constraints and allowing for a much longer and natural interaction (Rowe \& GoldinMeadow, 2009a). In fact, Rowe (2000) also did not find a relation between parent and infant total points in study that used a similar structured free-play to that in the current study. Further, Liszkowski and Tomasello (2011) only found a relation based on a dichotomized measures of parent and infant pointing. Previous studies have found evidence that the context influences parents language and gesture use (Puccini, Hassemer, Salomo, \& Liszkowski, 2010; Salo, Rowe, Leech, \& Cabrera, 2015), however the differences in findings across these studies could be driven by a variety of factors including sample size, age range of the infants, and/or methodology and more work is need to parse apart the various factors that might influence the relation between parents' and infants' pointing. We did find a trend in the relations between mothers' pointing and their infants' pointing when examined at the level of communicative intent. Mothers of infants who produced declarative points tended to produce more points and more declarative points themselves, as compared to mothers of infants who did not produce any declarative points. This relation seemed to be driven by mothers' expressive declarative points. However, these relations were not significant. As hypothesized, there were no relations between mother imperative points and infant pointing, suggesting that parents' production of points in the context of sharing attention and emotion are more supportive of infants' own use of points. 


\section{Relations between parent and infant pointing, and infant language}

We also examined how parents' and infants' pointing production compare as predictors of developing language skill when measured at the level of their different communicative intentions.

Parent pointing is related to infant language.-We found relatively strong relations between parent pointing, particularly declarative pointing, and infants' receptive language skills at 12-months, but these relations were not evident at 18- or 24-months. Further, mothers' imperative pointing was not related to the measures of infant language at any time point. While beyond the scope of the current study there are several possibilities that could explain the functional role of parents' declarative pointing. Perhaps observing their parent's emotional response to an object or event, often positive emotions like surprise or delight, creates a state of arousal in the infant that supports attention to the referent and an enhanced state of learning. Another possibility is the language that parents use in conjunction with their pointing gestures. Perhaps parents more often label the referent with declarative points than with imperative points. It is likely a combination of these two, that parents are providing a label at a time when the infant is aroused and attentive, that supports the infant's language development and specifically word learning. Future studies are required to test these possibilities.

Importantly, the relations between mothers' pointing and infants' pointing and language remained significant above and beyond the effect of mothers' speech - so it is not adequate to say that mothers who point more in this way also talk more and are perhaps providing more labels along with their points, which is supporting their infants' receptive vocabulary growth. The current data does not rule out that explanation and that pathway is undoubtedly also important, however this finding suggests that mothers' expressive declarative points have a unique impact on their infants' language development.

Infant pointing is related to infant language.-Infants' overall pointing production was also related to their later language skills, such that infants who pointed more during the 12-month interaction, had larger MCDI expressive scores at 18 months, and larger receptive and expressive Vineland scores at 24 months, controlling for their 12-month language scores. Further, infants' who produced declarative points tended also to have stronger language skills. Most notably, infants' declarative pointing status was related to their concurrent receptive vocabulary, and to their receptive and expressive language scores one year later, even when controlling for 12-month language. This replicates the robust findings in previous research (Colonnesi et al., 2010), and provides further evidence that declarative pointing in infancy is an early marker of readiness to communicate and to share ideas and information. Recent work suggesting a fundamental link between infants' declarative gestures and their joint attention skills provides support for this hypothesis (Salo et al., 2018). Work by Lucca and Wilbourn (2016) also suggests that infants' pointing indicates a readiness to learn new words, in particular labels for objects. A recent study by Lüke and colleagues (Lüke et al., 2017) compared 12-month-old infants' pointing according to communicative intention (imperative or declarative) and hand shape (index-finger or openhand) as predictors of 24-month vocabulary. Interestingly, it was found that hand shape 
(producing index-finger points rather than pointing with an open hand) was a better predictor of later language than communicative intention. However, the measure of declarative pointing was continuous (mean of declarative points produced per trial in a setting that was intended specifically to elicit declarative points), whereas in the current study the measure was dichotomous. In fact, almost half of the infants in that study did not produce any declarative points. More work is thus needed to fully understand the unique mechanism(s) linking early declarative pointing and language development.

Parent and infant points, together, predict infant language.-When considered together in a regression model predicting concurrent infant receptive vocabulary, mothers' expressive declarative points were a significant predictor above and beyond the effect of infant declarative pointing, and the interaction between mothers' and infants' pointing was also significant. Specifically, we see that the positive relation between mothers' expressive declarative pointing and their infants' concurrent receptive language was present only for those infants who were also producing declarative points themselves. Taking these results in sum, perhaps infants who see their parent(s) using a lot of points in the context of sharing attention, emotion, and information about objects or events are more likely not only to produce these kinds of points themselves, but are also better able to understand the points. This heightened ability to respond to and understand their parents' declarative points combined with exposure to a lot of these points appears to be an important mechanism in developing their language skills. Alternatively, this finding may suggest that mothers use pointing differently depending on their infant's language level. That is, perhaps parents produce declarative points in response to how much they think their infant understands. More work is needed to tease about the directionality of these effects between parent and infant pointing and infant language.

The relations between parenting and infant pointing and language development were limited to the 12-month time-point in this study. Thus, we may be seeing evidence a developmental trajectory wherein mothers' early declarative pointing supports both their infant's early word learning and perhaps provides a model for their infant to begin using points as well. As the infant learns the various communicative functions of points, their own production of points, and in particular their declarative points, predicts further language development, however the role of maternal pointing may not sustain as infants can handle more complex verbal input from their parents as their own verbal skills increase. It should be noted that the measures of infant language in the current study are all parent-report. Parent reporting of language skills is influenced by several factors which can bias reporting (Bennetts, Mensah, Westrupp, Hackworth, \& Reilly, 2016). One potential factor that may bias parents reporting in the current sample is parents own use of expressive declarative points which may lead to the report of larger vocabularies for their infants. Likewise, parents who see their infant producing many points may be similarly inclined to report larger vocabularies for their infants. That is, parents' overall impression of their infant's communicative ability, influenced by both the infant's use of gesture and spoken or receptive language, may be reflected in their reports of their infants' language skills. Future replications should utilize other means of measuring infant language, such as standardized lab-based measures or observed spontaneous language, to rule this possibility out. Researchers are just beginning to 
understand the mechanisms linking infant pointing with their language development (Lucca \& Wilbourn, 2016; Wu \& Gros-Louis, 2015), however this is the first study to explore parents' pointing with their young infants at the level of communicative intention and more work is needed to fully understand the developmental mechanisms linking parent and infant pointing.

\section{Conclusions}

Infants' production of declarative points is seen by many as an early indicator of their communicative ability, and the current study suggests the importance of examining the points that parents use with their infants at the level of communicative intent as well. Our findings suggest that parents' and infants' use of declarative points may have unique and interacting effects on infants' developing language skills. Those infants who produce declarative points earlier than their peers may be able to capitalize on the learning opportunities afforded by their parents' declarative points thus resulting in stronger language development. This study constitutes an important initial exploration of these relations, however, more work is needed to parse apart the directionality and replicability of these effects.

\section{Acknowledgements}

The research presented here was funded by NIMH grant MH 080759 to Pat Levitt and Nathan Fox. The first author also received support from the NICHD Training Program in Social Development Grant (NIH T32 HD007542) awarded to the Department of Human Development and Quantitative Methodology at the University of Maryland by the NICHD. We would like to thank T. Li, B. Mullan, K. Yoo, R. Bingaman, A. Kresse, J. Goldstein, J. Osher and K. Read for their help assisting in data collection and coding, and all of the participating children and parents for their help.

\section{References}

Bates E, Camaioni L, \& Volterra V (1975). The acquisition of performatives prior to speech. MerrillPalmer Quarterly of Behavior and Development, 21(3), 205-226.

Begus K, \& Southgate V (2012). Infant pointing serves an interrogative function. Developmental Science, 15(5), 611-617. 10.1111/j.1467-7687.2012.01160.x [PubMed: 22925509]

Behne T, Liszkowski U, Carpenter M, \& Tomasello M (2012). Twelve-month-olds' comprehension and production of pointing. British Journal of Developmental Psychology, 30, 359-375. 10.1111/ j.2044-835X.2011.02043.x [PubMed: 22882368]

Bennetts SK, Mensah FK, Westrupp EM, Hackworth NJ, \& Reilly S (2016). The Agreement between Parent-Reported and Directly Measured Child Language and Parenting Behaviors. Frontiers in Psychology, 7, 1710 10.3389/fpsyg.2016.01710 [PubMed: 27891102]

Brooks R, \& Meltzoff AN (2008). Infant gaze following and pointing predict accelerated vocabulary growth through two years of age: A longitudinal, growth curve modeling study. Journal of Child Language, 35, 207-220. 10.1017/S030500090700829X [PubMed: 18300435]

Camaioni L (1997). The emergence of intentional communication in ontogeny, phylogeny, and pathology. European Psychologist, 2(3), 216-225. 10.1027/1016-9040.2

Carpenter M, Nagell K, \& Tomasello M (1998). Social cognition, joint attention, and communicative competence from 9 to 15 months of age. Monographs of the Society for Research in Child Development, 63(4).

Cartmill EA, Demir ÖE, \& Goldin-Meadow S (2012). Studying Gesture In Hoff E (Ed.), Research Methods in Child Language: A Practical Guide (1st ed., pp. 208-225). Blackwell Publishing Ltd. 
Cochet H, \& Byrne RW (2016). Communication in the second and third year of life: Relationships between nonverbal social skills and language. Infant Behavior and Development, 44, 189-198. 10.1016/j.infbeh.2016.07.003 [PubMed: 27450099]

Cochet H, \& Vauclair J (2010). Pointing gestures produced by toddlers from 15 to 30 months: Different functions, hand shapes and laterality patterns. Infant Behavior \& Development, 33(4), 431-441. 10.1016/j.infbeh.2010.04.009 [PubMed: 20546910]

Colonnesi C, Stams GJJM, Koster I, \& Noom MJ (2010). The relation between pointing and language development: A meta-analysis. Developmental Review, 30, 352-366. 10.1016/j.dr.2010.10.001

de Villiers Rader N, \& Zukow-Goldring P (2012). Caregivers' gestures direct infant attention during early word learning: the importance of dynamic synchrony. Language Sciences, 34(5), 559-568. 10.1016/j.langsci.2012.03.011

Desrochers S, Morissette P, \& Ricard M (1995). Two perspectives on pointing in infancy In Moore C \& Dunham PJ (Eds.), Joint attention: Its origins and role in development (pp. 85-101). Hillsdale, NJ: Lawrence Erlbaum Associates.

Fenson L, Dale PS, Reznick JS, Bates E, Thal DJ, \& Pethick SJ (1994). Variability in early communicative development. Monographs of the Society for Research in Child Development, 59(5). 10.2307/1166093

Franco F, \& Butterworth G (1996). Pointing and social awareness: declaring and requesting in the second year. Journal of Child Language, 23(02), 307-336. 10.1017/S0305000900008813 [PubMed: 8936689]

Goldin-Meadow S (2007). Pointing sets the stage for learning language--and creating language. Child Development, 78(3), 741-745. 10.1111/j.1467-8624.2007.01029.x [PubMed: 17517001]

Goldin-Meadow S, Mylander C, de Villiers J, Bates E, \& Volterra V (1984). Gestural Communication in Deaf Children: The Effects and Noneffects of Parental Input on Early Language Development. Monographs of the Society for Research in Child Development, 49(3/4), 1 10.2307/1165838

Harris PL, \& Lane JD (2014). Infants Understand How Testimony Works. Topoi, 33(2), 443-458. 10.1007/s11245-013-9180-0

Hart B, \& Risley TR (1992). American Parenting of Language-Learning Children: Persisting Differences in Family-Child Interactions Observed in Natural Home Environments. Developmental Psychology, 28(6), 1096-1105. 10.1037/0012-1649.28.6.1096

Hart B, \& Risley TR (1995). Meaningful differences in the everyday experience of young American children. Baltimore: Paul H Brookes Publishing.

Huttenlocher J, Haight W, Bryk A, Seltzer M, \& Lyons T (1991). Early vocabulary growth: Relation to language input and gender. Developmental Psychology, 27(2), 236-248. 10.1037/0012-1649.27.2.236

Iverson JM, \& Goldin-Meadow S (2005). Gesture paves the way for language development. Psychological Science, 16(5), 367-371. 10.1111/j.0956-7976.2005.01542.x [PubMed: 15869695]

Knudsen B, \& Liszkowski U (2013). One-Year-Olds Warn Others About Negative Action Outcomes. Journal of Cognition and Development, 14(3), 424-436. 10.1080/15248372.2012.689387

Kovács ÁM, Tauzin T, Téglás E, Gergely G, \& Csibra G (2014). Pointing as Epistemic Request: 12month-olds Point to Receive New Information. Infancy, 19(6), 543-557. 10.1111/infa.12060 [PubMed: 26568703]

LeBarton ES, Goldin-Meadow S, \& Raudenbush S (2015). Experimentally Induced Increases in Early Gesture Lead to Increases in Spoken Vocabulary. Journal of Cognition and Development, 16(2), 199-220. 10.1080/15248372.2013.858041 [PubMed: 26120283]

Liszkowski U, Brown P, Callaghan T, Takada A, \& de Vos C (2012). A prelinguistic gestural universal of human communication. Cognitive Science, 36, 698-713. 10.1111/j.1551-6709.2011.01228.x [PubMed: 22303868]

Liszkowski U, Carpenter M, Henning A, Striano T, \& Tomasello M (2004). Twelve-month-olds point to share attention and interest. Developmental Science, 7(3), 297-307. 10.1111/ j.1467-7687.2004.00349.x [PubMed: 15595371]

Liszkowski U, Carpenter M, Striano T, \& Tomasello M (2006). 12- and 18-Month-Olds Point to Provide Information for Others. Journal of Cognition and Development, 7(2), 173-187. 10.1207/ s15327647jcd0702 
Liszkowski U, Carpenter M, \& Tomasello M (2008). Twelve-month-olds communicate helpfully and appropriately for knowledgeable and ignorant partners. Cognition, 108, 732-739. 10.1016/ j.cognition.2008.06.013 [PubMed: 18721918]

Liszkowski U, \& Tomasello M (2011). Individual differences in social, cognitive, and morphological aspects of infant pointing. Cognitive Development, 26(1), 16-29. 10.1016/j.cogdev.2010.10.001

Lock A, Young A, Service V, \& Chandler P (1990). Some observations on the origin of the pointing gesture In Volterra V \& Erting CJ (Eds.), From gesture to language in hearing and deaf children (pp. 42-55). Berlin: Springer-Verlag.

Lucca K, \& Wilbourn MP (2016). Communicating to Learn: Infants' Pointing Gestures Result in Optimal Learning. Child Development. 10.1111/cdev.12707

Lüke C, Grimminger A, Rohlfing KJ, Liszkowski U, \& Ritterfeld U (2017). In Infants’ Hands: Identification of Preverbal Infants at Risk for Primary Language Delay. Child Development, 88(2), 484-492. 10.1111/cdev.12610 [PubMed: 27562074]

MacWhinney B (2000). The CHILDES Project: Tools for analyzing talk, Volume II: The database (Vol. 2). Mahwah, NJ: Lawrence Erlbaum Associates.

Matthews D, Behne T, Lieven E, \& Tomasello M (2012). Origins of the human pointing gesture: a training study. Developmental Science, 15(6), 817-829. 10.1111/j.1467-7687.2012.01181.x [PubMed: 23106736]

Mundy P, Block J, Delgado C, Pomares Y, Van Hecke AV, \& Parlade MV (2007). Individual differences and the development of joint attention in infancy. Child Development, 78(3), 938-954. 10.1111/j.1467-8624.2007.01042.x [PubMed: 17517014]

Pan BA, Rowe ML, Singer JD, \& Snow CE (2005). Maternal correlates of growth in toddler vocabulary production in low-income families. Child Development, 76(4), 763-782. 10.1111/ j.1467-8624.2005.00876.x [PubMed: 16026495]

Puccini D, Hassemer M, Salomo D, \& Liszkowski U (2010). The type of shared activity shapes caregiver and infant communication. Gesture, 10(i), 279-296. 10.1075/gest.10.2-3.08puc

Reeb-Sutherland BC, Levitt P, \& Fox NA (2012). The Predictive Nature of Individual Differences in Early Associative Learning and Emerging Social Behavior. PLoS ONE, 7(1), e30511 10.1371/ journal.pone.0030511 [PubMed: 22291971]

Rowe ML (2000). Pointing and talk by low-income mothers and thier 14-month-old children. First Language, 20, 305-330. Retrieved from http://fla.sagepub.com/content/20/60/305.short

Rowe ML (2012). A longitudinal investigation of the role of quantity and quality of child-directed speech in vocabulary development. Child Development, 83(5), 1762-1774. 10.1111/ j.1467-8624.2012.01805.x [PubMed: 22716950]

Rowe ML, \& Goldin-Meadow S (2009a). Differences in early gesture explain SES disparities in child vocabulary size at school entry. Science, 323, 951-953. 10.1126/science.1167025 [PubMed: 19213922]

Rowe ML, \& Goldin-Meadow S (2009b). Early gesture selectively predicts later language learning. Developmental Science, 12(1), 182-187. 10.1111/j.1467-7687.2008.00764.x [PubMed: 19120426]

Rowe ML, \& Leech KA (2018). A parent intervention with a growth mindset approach improves children's early gesture and vocabulary development. Developmental Science, e12792 10.1111/ desc. 12792

SALO VC, ROWE ML, LEECH KA, \& CABRERA NJ (2015). Low-income fathers' speech to toddlers during book reading versus toy play. Journal of Child Language, 1-15. 10.1017/ S0305000915000550

Salo VC, Rowe ML, \& Reeb-Sutherland BC (2018). Exploring infant gesture and joint attention as related constructs and as predictors of later language. Infancy. 10.1111/infa.12229

Salomo D, \& Liszkowski U (2013). Sociocultural Settings Influence the Emergence of Prelinguistic Deictic Gestures. Child Development, 84(4), 1296-1307. 10.1111/cdev.12026 [PubMed: 23252681]

Sauer E, Levine SC, \& Goldin-Meadow S (2010). Early gesture predicts language delay in children with pre- or perinatal brain lesions. Child Development, 81(2), 528-539. 10.1111/ j.1467-8624.2009.01413.x [PubMed: 20438458] 
Southgate V, Van Maanen C, \& Csibra G (2007). Infant pointing: Communication to cooperate or communication to learn? Child Development, 78(3), 735-740. 10.1111/j.1467-8624.2007.01028.x [PubMed: 17517000]

Sparrow SS, Cicchetti DV, \& Balla DA (2005). Vineland adaptive behavior scales 2nd edition. Circle Pines, MN: American Guidance Service.

Tomasello M, Carpenter M, \& Liszkowski U (2007). A new look at infant pointing. Child Development, 78(3), 705-722. 10.1111/j.1467-8624.2007.01025.x [PubMed: 17516997]

Woodward AL, \& Guajardo JJ (2002). Infants' understanding of the point gesture as an object-directed action. Cognitive Development, 17(1), 1061-1084. 10.1016/S0885-2014(02)00074-6

$\mathrm{Wu} \mathrm{Z}$, \& Gros-Louis J (2015). Caregivers provide more labeling responses to infants' pointing than to infants' object-directed vocalizations. Journal of Child Language, 42(3), 538-561. 10.1017/ S0305000914000221 [PubMed: 24923871]

Yu C, \& Smith LB (2012). Embodied attention and word learning by toddlers. Cognition, 125(2), 244262. 10.1016/j.cognition.2012.06.016 [PubMed: 22878116] 


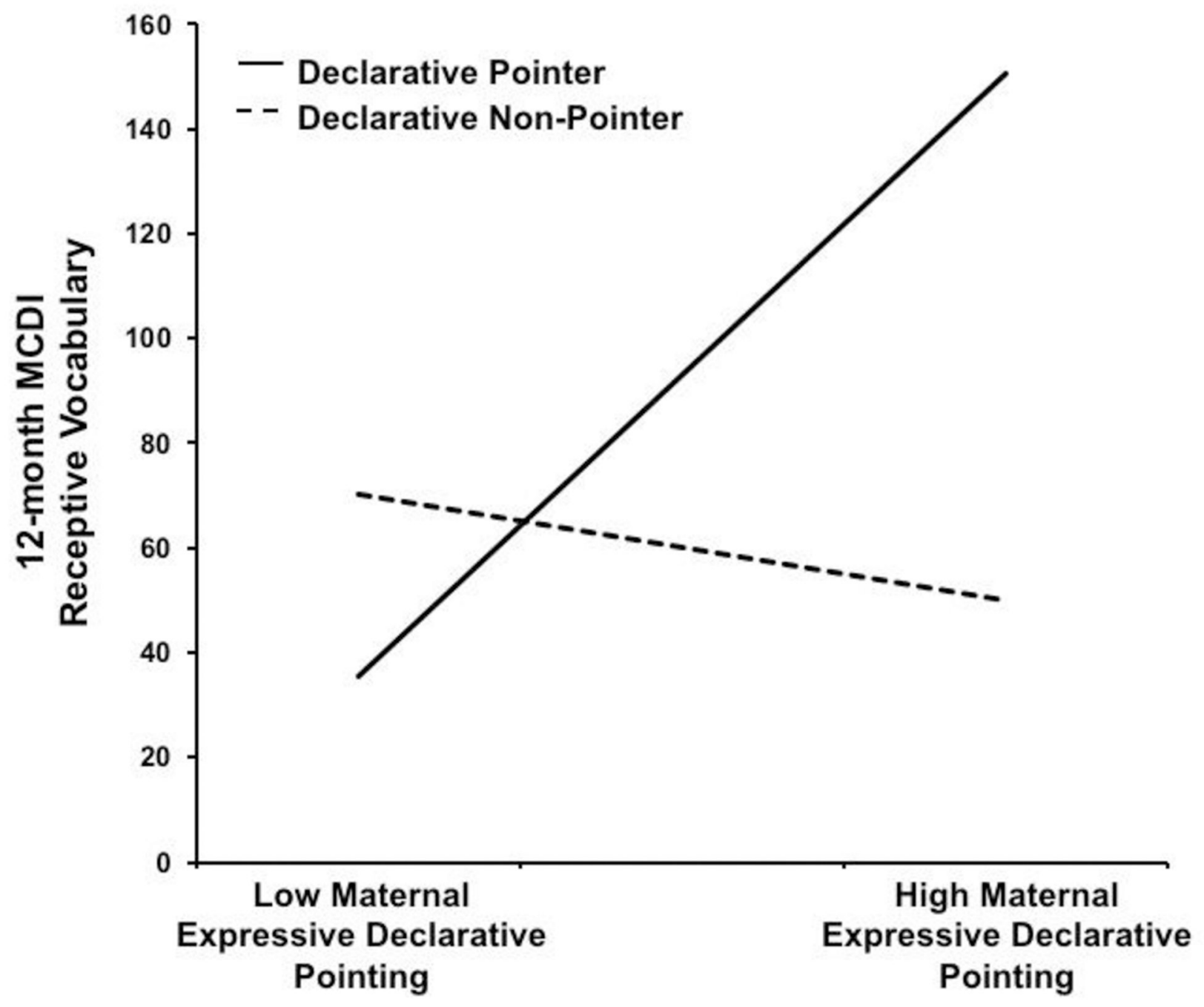

Figure 1.

Significant effects of mothers' expressive declarative pointing on concurrent child receptive vocabulary qualified by infants' declarative pointing status. Low and high scores correspond to $-1 \mathrm{SD}$ below and $+1 \mathrm{SD}$ above the mean. 
Table 1.

Descriptive statistics for parent and infant pointing

\begin{tabular}{lll}
\hline & Mean (SD) & Range \\
\hline Infant pointing & & \\
\multicolumn{1}{c}{ Total points } & $2.17(3.29)$ & $0-14$ \\
$\quad$ Declarative points & $1.27(2.19)$ & $0-10$ \\
$\quad$ Expressive declarative & $1.25(2.20)$ & $0-10$ \\
$\quad$ Informative declarative & $0.02(0.14)$ & $0-1$ \\
& & \\
Mother pointing & & \\
Total points & $20.92(14.67)$ & $0-68$ \\
$\quad$ Imperative points & $7.63(7.50)$ & $0-41$ \\
$\quad$ Declarative points & $13.21(10.26)$ & $0-53$ \\
$\quad$ Expressive declarative & $8.48(7.12)$ & $0-35$ \\
$\quad$ Informative declarative & $4.73(4.96)$ & $0-21$ \\
\hline
\end{tabular}

Note. $\mathrm{N}=52$. 
Table 2.

Mother pointing production compared across infant declarative pointing status

\begin{tabular}{llllllll}
\hline Mother Pointing & \multicolumn{7}{l}{ Infant 12-month Declarative Pointing Status } \\
& \multicolumn{2}{l}{ Did produce } & \multicolumn{2}{c}{ Did not produce } & $\boldsymbol{t}$ value & $\boldsymbol{p}$ value \\
& M & SD & M & SD & & \\
\hline Total Points & 24.00 & 16.91 & 18.07 & 11.86 & 1.47 & .15 \\
Imperative points & 8.00 & 6.97 & 7.30 & 8.08 & 0.34 & .74 \\
Declarative points & 15.96 & 11.76 & 10.67 & 8.07 & 1.91 & .06 \\
$\quad$ Expressive declarative & 10.28 & 7.79 & 6.81 & 6.12 & 1.79 & .08 \\
$\quad$ Informative declarative & 5.68 & 6.10 & 3.85 & 3.49 & 1.34 & .19 \\
\hline
\end{tabular}

Note: $\mathrm{d}=50$ 
Table 3.

Infant language scores compared across infant declarative pointing status

\begin{tabular}{|c|c|c|c|c|c|}
\hline \multirow[t]{3}{*}{ Infant Language } & \multicolumn{5}{|c|}{ Infant 12-month Declarative Pointing Status } \\
\hline & \multicolumn{2}{|c|}{ Did produce } & \multicolumn{2}{|c|}{ Did not produce } & \multirow[t]{2}{*}{$t$ value } \\
\hline & M & SD & M & SD & \\
\hline 12-month MCDI receptive & 105.30 & 87.42 & 62.26 & 50.96 & $2.04 *$ \\
\hline 12-month MCDI expressive & 7.87 & 7.00 & 6.74 & 6.38 & .572 \\
\hline 18-month MCDI receptive & 237.78 & 90.83 & 190.71 & 87.27 & $1.75^{\dagger}$ \\
\hline 18-month MCDI expressive & 92.74 & 66.47 & 60.62 & 53.71 & $1.75^{\dagger}$ \\
\hline 24-month MCDI expressive & 418.55 & 162.66 & 337.64 & 163.15 & 1.66 \\
\hline 24-month Vineland receptive & 17.17 & 2.17 & 16.16 & 1.41 & $1.86^{\dagger}$ \\
\hline 24-month Vineland expressive & 16.95 & 1.13 & 16.04 & 1.46 & $2.38^{*}$ \\
\hline
\end{tabular}

Note. For 12-month MCDI n=46, df=44, for 18-month MCDI $\mathrm{n}=44, \mathrm{df}=42$, and for 24-month MCDI and Vineland $\mathrm{n}=47$, $\mathrm{df}=45$.

p $<.05$

$t_{\mathrm{p}}<.10$. 


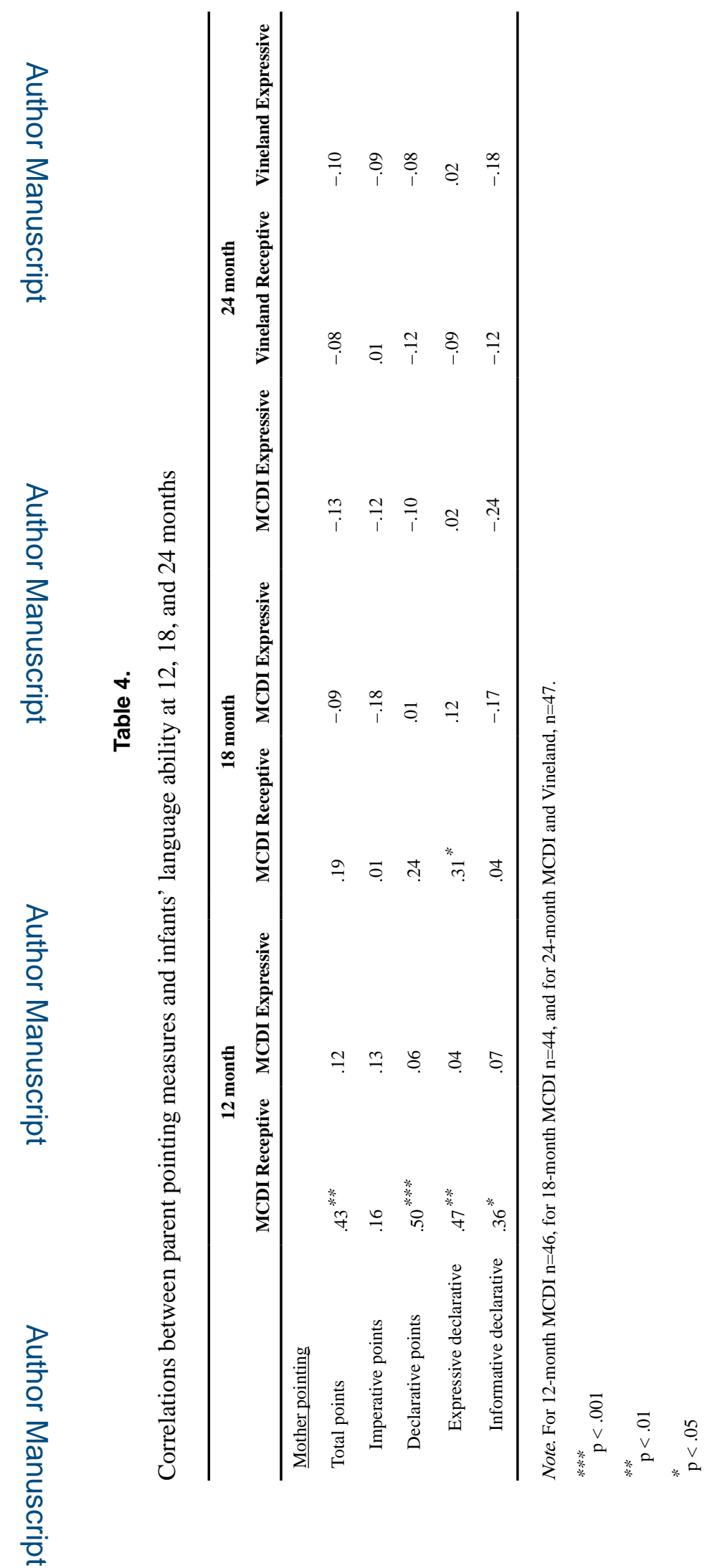

J Cogn Dev. Author manuscript; available in PMC 2020 February 21. 
Table 5.

Regression models predicting infant receptive vocabulary at 12-months

\begin{tabular}{|c|c|c|c|c|}
\hline & \multicolumn{4}{|c|}{ Receptive Vocabulary (MCDI) B (se) } \\
\hline & Model 1 & Model 2 & Model 3 & Model 4 \\
\hline Intercept & $\begin{array}{l}84.07^{* * *} \\
(9.77)\end{array}$ & $\begin{array}{l}83.78^{\text {*** }} \\
(10.55)\end{array}$ & $\begin{array}{l}81.817^{* * * *} \\
(9.65)\end{array}$ & $\begin{array}{l}75.07^{* * * *} \\
(8.63)\end{array}$ \\
\hline Mother expressive declarative points & $\begin{array}{l}4.85^{* *} \\
(1.39)\end{array}$ & & $\begin{array}{l}4.27^{* *} \\
(1.36)\end{array}$ & $\begin{array}{l}3.24^{*} \\
(1.22)\end{array}$ \\
\hline Infant declarative pointing status & & $\begin{array}{l}43.04^{*} \\
(21.10)\end{array}$ & $\begin{array}{l}29.67 \\
(19.73)\end{array}$ & $\begin{array}{l}28.65 \\
(17.26)\end{array}$ \\
\hline Mother $\times$ Infant & & & & $\begin{array}{l}9.23^{* *} \\
(2.45)\end{array}$ \\
\hline $\mathrm{R}^{2} \%$ & 21.7 & 8.6 & 25.6 & 44.4 \\
\hline F stat & $12.19^{* *}$ & $4.162 *$ & $7.40^{* *}$ & $11.19^{* * *}$ \\
\hline
\end{tabular}

Note. $\mathrm{N}=46$.

**** $p<.001$

*** $p<.01$

* $p<.05$ 